\title{
PAPER
}

\section{Effects of left parietal injury on covert orienting of attention}

\section{U Castiello, M Paine}

J Neurol Neurosurg Psychiatry 2002;72:73-76

See end of article for authors' affiliations

\section{Correspondence to:}

Dr U Castiello, Department of Psychology, Royal Holloway, University of London, Egham, Surrey TW20 OEX, UK; u.castiello@rhul.ac.uk

Received 18 April 2001 In revised form 6 July 2001 Accepted 6 July 2001
Objective: To assess the effects of left parietal injury on covert visual attention during a detection task and a pointing task.

Methods: The Posner's paradigm was given to a patient who was found at the age of 74 to have spent all his life without the left parietal lobe as a result of a congenital perinatal insult and to a control subject. In one session subjects were required to provide an arbitrary response at stimulus appearance (key press). In another session subjects were required to point to the stimulus.

Results: The patient was able to disengage covert attention from a cued position when the task was to provide an arbitrary key press response in a similar fashion to a control subject with no neurological deficits. By contrast, he was impaired in disengaging attention from a cued position when the task was to reprogramme an overt pointing action.

Conclusions: Response to cued information is differentially available depending on task. It is suggested that mechanisms concerned with the attention for action systems are located within the left parietal lobe. n the present study we assessed the covert orienting of attention in a patient with a left parietal lesion by applying a standard experimental procedure. ${ }^{1}$ Covert orienting of attention is achieved in the absence of explicit eye or body movements, and it has been well characterised by the works of Posner. ${ }^{1}$ In the Posner paradigm, subjects are required to respond as quickly as possible to stimuli which are presented either in expected (valid condition), equally probable (neutral condition) and unexpected (invalid condition) positions of the visual field. Benefits to reaction time (quicker responses) are usually found for the valid condition while costs (slower responses) are usually found for the invalid condition. Comparisons of these benefits and costs are thought to provide an indication of the orienting efficiency of covert attention. This paradigm allows assessment of the dissociable functions of orienting and disengaging attention. Relating the

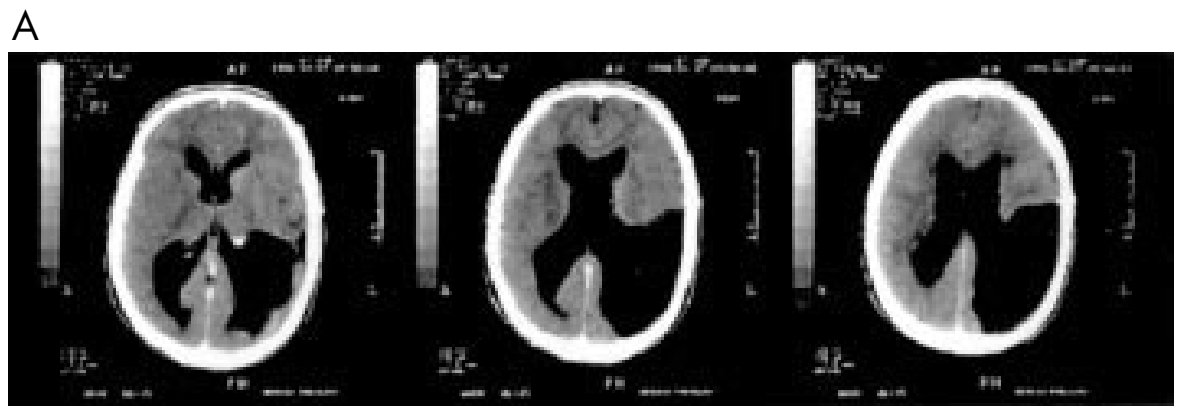

B
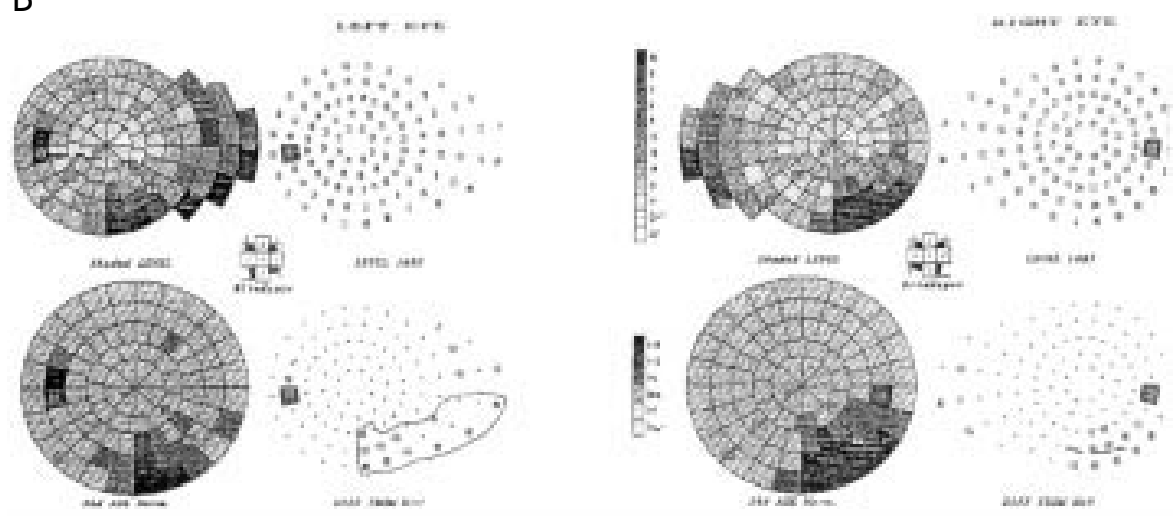

Figure 1 (A) Brain CT shows a large area of porencephaly almost obliterating the parietal lobe on the left (the left hemisphere is shown on the right side of the image). (B) Automated perimeter for the left and the right eye. 
anatomy of pathology to the aforementioned elements has promoted the formulation of hypotheses as to the neural substrates of covert attentional functions.

Recently, the notion of covert orienting has been extended not only to processes concerned with orienting or eye movements but to an analogous process associated with the preparation of limb movements. ${ }^{2}$ Rushworth et al ${ }^{2}$ developed a task where precues allow subjects to covertly prepare for hand movements as opposed to covertly preparing for orienting, or eye movement. They refer to this process as motor attention to distinguish it from orienting attention. These authors tested a group of subjects with lesions confined within the left parietal cortex and a group of subjects with lesions confined within the right parietal cortex. It was found that both groups showed the same ability to engage attention to a movement when the cue was valid. However, participants within the group having a left parietal lesion were impaired in their ability to disengage the focus of motor attention when the precue was invalid. In the light of these results the authors proposed a left hemisphere dominance for motor tasks or at least for tasks that require greater attention to action.

We capitalise on these known effects to further explore the idea of a covert "motor" attentional system located within the left parietal lobe by testing a patient without the left parietal lobe.

\section{MATERIALS AND METHODS \\ Participants}

\section{Case history}

A 74 year old left handed man, was found to have an incomplete right homonymous hemianopia during the evaluation of glaucoma. Brain CT unexpectedly disclosed a large area of porencephaly almost totally obliterating the left parietal lobe, which was most likely to have been sustained as a result of perinatal insult (fig 1 A). An automated perimeter (fig l B) shows that the field defect was quite subtle to confrontation-a homonymous inferior sector aligned to the vertical meridian.

\section{Neuropsychological examination}

Administration of the Wechsler adult intelligence scalerevised showed a full scale IQ of 81 , with a verbal IQ of 81 and a performance IQ of 84 . He also performed within normal limits on tests of verbal and visual memory. His only significant impairment was on a task requiring him to generate words beginning with a given letter. Visuoperceptual function was intact. He was able to copy both simple and more complex figures and draw from memory. He was also able to recognise and name pictures of familiar objects. There was no evidence of ideomotor or ideational apraxia. Furthermore, no evidence of neglect was found on line bisection tasks, cancellation tasks or copying tasks. The patient was also checked for extinction by using an extinction task where unilateral and bilateral stimuli, with catch trials interspersed, were presented. No extinction was found. A control subject matched for age, sex, and handedness was also tested. The control subject reported no neurological or skeletomotor dysfunctions. The subjects took part in two experimental sessions conducted on two separate days.

\section{Reaction time task}

The experimental set up is represented in figure 2. Participants were seated in front of a computer screen (20") driven by Pentium computer. The head was placed in a head and chin rest which was adjusted so that a distance of $50 \mathrm{~cm}$ stretched between the participants' eyes and the computer screen. The eyes were level with the centre of the computer screen. Two sets of infrared phototransistor sensors were attached to the head and chin rest as part of a system for controlling vertical and horizontal eye movements (ASL-210). Vertical movements greater than $0.5^{\circ}$ of visual angle from a central gaze fixation and horizontal movements greater than $0.25^{\circ}$ of visual angle from a central gaze fixation point were automatically detected and the corresponding trial was discarded and replaced. A microswitch was used to record reaction times.

The visual display consisted of two boxes $(6 \times 6 \mathrm{~cm}$, fig 2) presented $10^{\circ}$ to the right or to the left of a white fixation point ( $1 \mathrm{~cm}$ diameter). The squares were light blue-green in colour with a luminance of $34 \mathrm{~cd} / \mathrm{m}^{2}$ presented against a black background of zero luminance.

To direct covert attention to one of the two boxes, the boxes briefly flashed. The flash was the cue to inform the subjects in which of the two squares the stimulus was likely to appear. The cue was followed by a target stimulus in the form of a red circle (diameter $2.5 \mathrm{~cm}$ ) presented at the centre of the square (fig 2). The stimulus had a luminance of $16 \mathrm{~cd} / \mathrm{m}^{2}$

\section{Procedure}

An initial calibration procedure preceded each experimental session. During this period the participant was seated and the eye movement monitoring equipment was positioned appropriately. Each trial began with the appearance of two squares and a fixation dot displayed in the centre of the screen. The fixation dot and the two squares remained for the duration of the trial. Participants were instructed to first wait for the appearance of the cue, and then to use this cue as a guide to which square the stimulus would appear. Participants were asked to respond as quickly as possible by pressing the button with their left index finger as soon as the stimulus appeared.

After a constant delay of $500 \mathrm{~ms}$ after the appearance of the squares, the cue was presented for $250 \mathrm{~ms}$. Directly after the offset of the cue there was an interval of $250 \mathrm{~ms}$, then the stimulus was presented and remained present until the response was emitted. The end of the trial was taken as either the time of response emission or $2000 \mathrm{~ms}$ after the stimulus presentation if no response was made. Participants were asked not to blink during each trial. The time of each trial was of sufficiently low duration for a blinkless period required for accurate eye movement detection. However, after the completion of each trial a message of "BLINK" was displayed to prevent the eyes becoming too fatigued.

Each participant first completed 20 practice trials followed by two blocks of 50 trials each, for a total of 100 experimental trials. The duration of each block was no longer than $10 \mathrm{~min}-$ utes, and all blocks were separated by a 10-20 minute rest period. All trials on which the following errors occurred were automatically reset to the end of the block to be re-presented randomly; errors due to eye movements, errors of anticipation

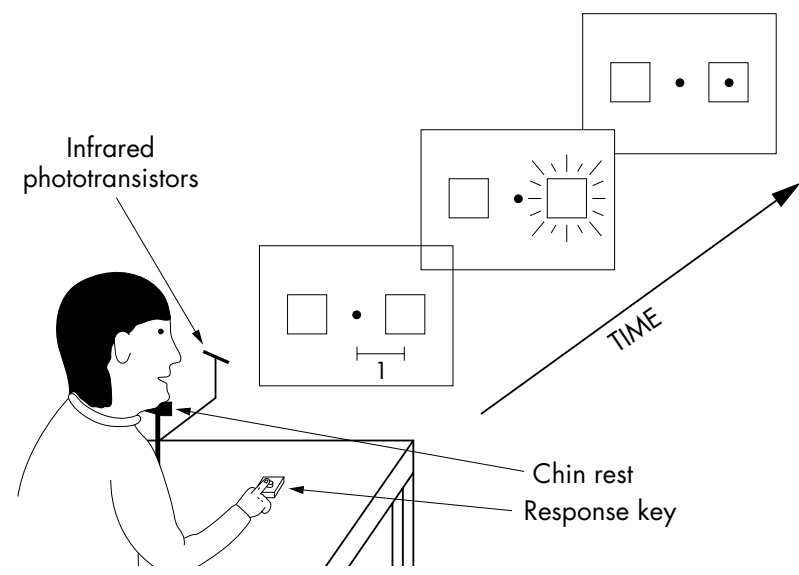

Figure 2 Experimental set up and sequence of events presentation The figure represents an example for valid trials. 
Table 1 Mean (SD) values for the dependent measures analysed for the reaction time and the pointing experiments

\begin{tabular}{|c|c|c|c|c|c|c|c|c|}
\hline & \multicolumn{4}{|l|}{ Patient } & \multicolumn{4}{|c|}{ Control participant } \\
\hline & \multicolumn{2}{|l|}{ Valid } & \multicolumn{2}{|l|}{ Invalid } & \multicolumn{2}{|l|}{ Valid } & \multicolumn{2}{|l|}{ Invalid } \\
\hline & Left & Right & Left & Right & Left & Right & Left & Right \\
\hline $\begin{array}{l}\text { Reaction time experiment } \\
\text { Pointing experiment }\end{array}$ & $471(85)$ & $473(76)$ & $521(85)$ & $528(78)$ & $458(65)$ & $463(79)$ & $509(67)$ & $513(77)$ \\
\hline Reaction time (ms) & $465(72)$ & $454(60)$ & $556(75)$ & $560(78)$ & 462 (69) & $459(74)$ & $509(86)$ & $510(80)$ \\
\hline Movement duration (ms) & $525(75)$ & $522(83)$ & $601(85)$ & 595 (82) & $520(76)$ & $517(76)$ & 541 (72) & $545(76)$ \\
\hline
\end{tabular}

(reaction time $<150 \mathrm{~ms}$ ), non-responses (or those made over $2000 \mathrm{~ms}$ ). Three types of trials were presented: (a) valid trials in which the cue and stimulus both appeared in the same square; (b) invalid trials in which the cue and stimulus appeared in different squares; (c) catch trials occurred when the cue, but not the stimulus would appear. These were included to further prevent expectancy or practice effects. The percentages of trials were as follows: valid trials (70\%), invalid trials $(20 \%)$, and catch trials $(10 \%)$. The order of these trials according to trial type was randomised within each block.

\section{Pointing task}

Methods and procedure were similar to those utilised for the reaction time task except that the participants were required to point with the index finger of their dominant hand as fast as possible to the location indicated by the cue instead of pressing a key. The duration of pointing movements was recorded with the ELITE motion analysis system. ${ }^{3}$ The subjects wore a marker attached to the index finger (the radial side of the nail).

\section{Data analysis}

To have the same number of trials for valid and invalid trials, 20 valid trials ( 10 right, 10 left) were chosen randomly from the pool of the collected valid trials. For the reaction time experiment mean reaction times were submitted to a repeated measures analysis of variance (ANOVA) with participant (patient, control) as a between subjects factor. Type of trial (valid, invalid) and visual field (right or left) were the within subjects factors. A similar analysis was conducted for the dependent measures considered for the pointing experiment: (1) reaction time was calculated from the moment the stimulus appeared and the participants raised their index finger from the starting switch; (2) movement duration was calculated as the time when the index finger was raised from the starting switch and the time the index finger touched the screen.

\section{RESULTS}

\section{Reaction time task}

As shown in table 1 , reaction times for valid trials were faster than for invalid trials (main effect type of trial; $F(1,19)=54.05$, $\mathrm{p}<0.0001$ ). This result demonstrates that the patient and the control participant were able to engage attention to a location when they were forewarned by a valid precue. In particular, the costs associated with having oriented attention on the wrong part of the visual field were similar for both subjects. The difference between mean reaction times for valid and invalid trials was $53 \mathrm{~ms}$ for the patient and $50 \mathrm{~ms}$ for the control subject. No differences were found between stimulus side of presentation. No interactions between the participants, the type of trial, and position were found. The effect of fatigue was examined by performing an ANOVA on the mean reaction time values with participant (patient, control) as a between subjects factor and block $(1,2)$ as a within subject factor.
There was no effect of block $(p>0.05)$ and no significant interactions between block and participants.

\section{Pointing task}

As disclosed by the significant interaction between participant and type of trial $(F(1,19)=21.32, \mathrm{p}<0.0001$; see table 1$)$ the time to initiate the movement suggests that the patient performed significantly different from the control subject when an invalid cue made them prepare the wrong response. In other words, the patient had more difficulty in initiating the movement than the control subject in the condition where an invalid precue makes them initially prepare to make the wrong movement. For the patient the time to initiate the movement increased by $98 \mathrm{~ms}$. For the control subject this increase was $48 \mathrm{~ms}$. The same trend was found for movement duration (interaction participant by type of trial; $F(1,19)=12.32, \mathrm{p}<0.001)$. As reported in table $\mathrm{l}$, for both subjects movement duration was longer for invalid than for valid trials. However, this increase in movement duration was more accentuated for the patient than for the control subject ( $74 \mathrm{~ms}$ $v 24 \mathrm{~ms} ; \mathrm{p}<0.01$ ). The effect of fatigue was examined by performing an ANOVA on the mean reaction time and movement duration values with the participant (patient, control) as a between subjects factor and block $(1,2)$ as a within subject factor. There was no effect of block $(p>0.05)$ and no significant interactions between block and participants. In summary, these results show that the patient and the control participant were able to engage attention to a location when they were forewarned by a valid precue even when an overt pointing action was required. However, the patient took longer to initiate and complete the pointing action than the control subject after an invalid cue. No differences were found for stimulus side of presentation (see table 1).

\section{DISCUSSION}

The purpose of the present study was to investigate cerebral dominance for a covert attentional mechanism concerned with overt action. Results provide further support for a covert orienting of attention mechanism concerned with overt action guidance located within the left parietal lobe. ${ }^{2}{ }^{4}$ The patient is similar to the control subject in being able to engage visual and motor covert attention when there is a valid precue for both the reaction time and the pointing tasks. He shows similar costs as the control subject after invalid precue for the reaction time task. However, he shows a significantly greater cost than the control subject after invalid precue for the pointing task.

An influential and controversial theoretical framework within the current attentional literature is the premotor theory of attention. ${ }^{5}$ According to the premotor model a covert shift of attention involves the same neural circuits as those involved in programming an overt eye movement or an arm movement. ${ }^{5}$ Further, another main proposition that characterises this model is that space is represented in several pragmatic maps. Some of these maps control oculomotion, others control movement of the arms and other body parts. To 
date, however, a definite answer to the question of whether covert spatial attention is exclusively related to oculomotion, as results from the classic Posner paradigm seems to suggest, or whether it is a mechanism resulting from other nonoculomotor pragmatic maps cannot be given. Nevertheless, the results of the present study provide some new insights to clarify this issue. Firstly, they suggest that the preparation to reach an object improves the capacity to select a location in the same way as the preparation to make a saccade does. Secondly, the maps controlling covert attention with respect to an overt limb action seem to be concentrated within the left parietal lobe. The patient was able to engage and disengage the focus of visual attention in a similar fashion as the control subject when no pointing action was required. When pointing was required this participant shows problems in initiating and implementing the action to a new position. Why is it difficult for the patient to reorganise the pointing action towards a new location in space after an invalid cue? Picking up on the notion of pragmatic maps raised within the context of the premotor theory of attention, a possible explanation is that only preparation maps to control for oculomotion are active for the reaction time task, whereas for the pointing task preparation maps to control for oculomotion and for movement of the arm are activated simultaneously. Along these lines, if "motor" attention is a predominant function of the left parietal cortex ${ }^{2}$ whereas oculomotor attention is a more predominant function of the right posterior parietal cortex, ${ }^{6}$ the effect found for the patient might be the result of the limited number of remaining maps concerned with movement programming and execution contained within the right parietal lobe.

The results for the patient also allow speculations on attentional functions carried out by the left and the right parietal lobes in terms of task relevance. ${ }^{7}$ When detection is the task, the patient's performance is similar to that of the control subject because such an arbitrary response does not require the planning of movement trajectory and selection of action has not been performed. The spatial task is thus confined to detection of the stimulus position. To this end the patient's intact right parietal lobe allows a performance which is similar to the performance you would expect for the classic Posner paradigm. ${ }^{1}$ It is well known that the right posterior parietal cortex has a pre-eminent role in this form of attention in both ipsilateral and contralateral directions. ${ }^{6}$ By contrast, when pointing was the task, the patient had to try to use left parietal lobe mechanisms that were not there. ${ }^{28}$ Thus, he had to rely on the compensatory functions of the right parietal lobe that possibly are not as efficient as the left parietal lobe in the updating of sensorimotor maps during overt actions. The delay in movement execution might thus be the result of a sequential implementation of functions within the only parietal lobe available to the patient rather than a parallel use of attentional functions carried out by both the left and the right parietal lobes.
In conclusion, the present results confirm the proposed role played by the left parietal cortex in the preparation of selected movements. ${ }^{8-10}$ The type of dissociation we have found in the patient seems to allow speculations about the asymmetric motor attentional system concerned with the preparation of hand action that is independent of preparation for an eye movement. A similar dissociation in patients with left parietal lesions has been found by Rushworth et al. ${ }^{2}$ Similarly to the present results, patients with left parietal lesions were slow to respond in a motor task when the precue was invalid-that is, when the cue specified the finger opposite to the one they were preparing to move on the basis of the precue. The present study enlarges the notion of the left hemisphere dominance for motor attention proposing a role for the left parietal lobe also for the reprogramming and execution of an overt action.

\section{ACKNOWLEDGEMENTS}

This work was supported by an NH-MRC grant to UC. Dean Lusher is thanked for his assistance. Anne Castles is thanked for conducting the neuropsychological assessment. We also thank the patient and his wife for their collaboration and for coming to the laboratory so many times.

\section{Authors' affiliations}

U Castiello, Department of Psychology, Royal Holloway, University of London, Egham, Surrey TW20 OEX, UK

U Castiello, M Paine, Department of Clinical Neuroscience, St Vincent's Hospital, Melbourne, Australia

M Paine, Neuro-Ophthalmology Clinic, Royal Victorian Eye and Ear Hospital, Melbourne, Australia

\section{REFERENCES}

1 Posner MI. Orienting of attention. The Vllth Sir Frederic Bartlett lecture. Q J Exp Psychol 1980;32:3-25.

2 Rushworth MFS, Nixon PD, Renowden S, et al. Neuropsychologia 1997;35: 1261-73.

3 Ferrigno G, Pedotti A. ELITE: a digital dedicated hardware system for movement analysis via real-time tv-signal processing. IEEE Trans Biomed Eng 1985;32:943-50

4 Rushworth M, Ellison A, Walsh V. Complementary localization and lateralization of orienting and motor attention. Nat Neurosci 2001:4:656-61.

5 Rizzolatti G, Riggio L, Sheliga BM. Space and selective attention. In: Umilta C, Moscovitch M, eds. Attention and performance. Vol XV. Conscious and nonconscious processing. Cambridge, MA: MIT Press, 1994:231-65.

6 Corbetta M, Miezin FM, Shulman GL, et al. A PET study of visuospatial attention. J Neurosci 1993;13:1202-26.

7 Castiello U. The effect of abrupt onset of 2D and 3D distractors on prehension movements. Percept Psychophys 2001;63:1014-25.

8 Schluter ND, Krams M, Rushworth MFS, et al. Cerebral dominance for action in the human brain: the selection of actions. Neuropsychologia 2000;39:105-13.

9 Deiber MP, Ibanez V, Sadato N, et al. Cerebral structures participating in motor preparation in humans: a positron emission tomography study. $J$ Neurophysiol 1996;75:233-46.

10 Krams M, Rushworth M, Deiber MP, et al. The preparation, execution and suppression of copied movements in the human brain. Exp Brain Res 1998; 120:386-98. 